\title{
Signal Coding and Interference Cancellation of Spectrally Efficient FDM Systems for 5G Cellular Networks
}

\author{
(Invited Paper) \\ Hedaia Ghannam and Izzat Darwazeh \\ Department of Electrical and Electronics Engineering \\ University College London \\ London, United Kingdom \\ E-Mail: hedaia.ghannam.15@ucl.ac.uk, i.darwazeh@ucl.ac.uk
}

\begin{abstract}
This paper explores new multicarrier signals and systems for 5G; spectrally efficient frequency division multiplexing (SEFDM), in which higher spectral efficiency (SE) compared to conventional orthogonal frequency division multiplexing (OFDM) is achieved by violating the orthogonality of its subcarriers. This work proposes new system and receiver models and then investigates the employment of various forward error correction (FEC) techniques, as well as a new interference cancellation receiver architecture to improve the overall system performance by ameliorating the effects of inter-carrier interference (ICI). Results show that the use of coded SEFDM system can drastically increase the SE by up to $67 \%$ relative to OFDM, at the expense of a power penalty below $3 \mathrm{~dB}$.
\end{abstract}

\section{INTRODUCTION}

Currently, 5G (IMT-2020) wireless technology is one of the central themes in International Telecommunications Union (ITU) research groups and many industry forums, with the goal being commercially deployed around 2020 as the name suggests. The $5 \mathrm{G}$ network promises to deliver the gigabit experience to mobile users, with ultra reliable and low latency communications, besides massive low rate machine type communications and lower energy consumption [1].

The special interest in orthogonal frequency division multiplexing (OFDM) for 4G LTE cellular network standards was mainly motivated by its spectrum structure with overlapping subcarriers, which not only enhances bandwidth efficiency but also significantly improves immunity against multipath propagation effects, when compared to single carrier transmission. In addition, the ease of implementation of OFDM transmitters and receivers using IFFT and FFT blocks made it attractive for a wide variety of applications [2] [3].

The advantages of OFDM made it (and its variants) key technologies for the physical layer of 5G [2]. A key question is: Can the spectral efficiency of OFDM be improved without sacrificing the key advantages of OFDM? One contribution in this direction was the development of spectrally efficient frequency division multiplexing (SEFDM) firstly proposed in 2003 [4]. SEFDM is a multi-carrier system that achieves spectral efficiency gains by packing the subcarriers closer (relative to OFDM), while compromising the orthogonality. SEFDM symbols are generated in a similar manner of OFDM using modified IFFT structures [5], yet they require more complex receiver structures [6]. Despite the non-orthogonality, different detection methods have been demonstrated where the error performance of SEFDM is reasonably close to OFDM, with SE improvement greater than $25 \%$ [7].

The multi-stream faster than Nyquist (FTN) technique proposed in [8], is SEFDM's time domain counterpart and has similar spectral efficiency gains with little error performance loss relative to OFDM. Other modulation candidates for $5 \mathrm{G}$ system with improved SE have been analyzed and compared in [9]. The substantial motivation behind all of these techniques is to minimize the out of band emission (OOBE), or in other words, improve the carrier to interference ratio (CIR) to reduce overhead and improve spectrum utilization. This may be achieved by windowing the symbols in time to smooth the transition between adjacent symbols; such as in windowed OFDM (W-OFDM), or filtering a bank of subcarriers to reduce the spectrum leakage. Filtered-OFDM (f-OFDM) and universal filtered multicarrier (UFMC) are two popular methods along this line. A more sophisticated/ complex filtering on the subcarrier level such as filter bank multi-carrier (FBMC) and generalized frequency-division multiplexing (GFDM) represent other candidates for $5 \mathrm{G}$.

In [10], SE improvement of $13 \%$ is reported for QAMFBMC system compared to OFDM and the results of [9] indicate that f-OFDM leads other filtering methods by an SE gain of $33 \%$ compared to cyclic prefixed OFDM (CP-OFDM).

Techniques commonly used in wireless systems, such as channel coding, and channel estimation and equalization have been applied to SEFDM with modification and have led to systems where significant spectral efficiency gains were reported in experimental wireless [7] and optical/mm-wave test beds [11]. As for ICI in SEFDM, its effects are similar to those encountered in OFDM system undergoing sever ICI. Therefore, the methods used for ICI mitigation of OFDM should apply, in principle, to SEFDM. Numerous techniques 
have been developed in recent years to overcome ICI, such as successive-interference cancellation (SIC) technique in GFDM, which is similar to a simple repetition channel coding where the information is not modulated onto just a single subcarrier but on two, to mitigate the effect of ICI [12]. Another technique is adding null (unused) subcarriers between a bank of OFDM symbols to eliminate ICI and ISI [13]. Furthermore, forward error correction (FEC) channel coding, commonly used in all cellular systems, is capable of reducing the errors caused by ICI at the expense of the addition of redundancy and therefore the reduction of effective spectral efficiency [14]. the efficacy of coupling interference cancellation with convolutional channel coding has been demonstrated for FTN in [8] and SEFDM in [15]. Coupling interference cancellation with a better channel coding, such as turbo coding, would be expected to result in further improved SEFDM performance and is the focus of this work.

The outline of this paper is as follows; we start with a short introduction to SEFDM signals in section II. The utilization of different channel coding techniques is investigated to improve the performance of SEFDM systems in section III. Section IV describes a new receiver architecture that combines the use of turbo coding and iterative interference cancellation and shows, through simulations, the efficacy of combining these two techniques. Finally, the conclusion is drawn in section V.

\section{SEFDM WAVEFORM DESIGN PROPERTIES}

\section{A. Discrete Time Model}

SEFDM is a multicarrier modulation technique, where symbols are generated similarly to OFDM as shown in [5], by means of modified IFFT structures. The equation below represents the discrete time domain $k^{\text {th }}$ SEFDM symbol at the transmitter side [5]

$$
X[k]=\frac{1}{\sqrt{Q}} \sum_{n=0}^{N-1} z_{n} \exp \left(\frac{j 2 \pi n k \alpha}{Q}\right)
$$

where the complex QAM baseband symbols $\mathbf{z}=\left\{z_{1}, z_{2}, \ldots z_{N}\right\}$ are modulated over $N$ subcarriers and sampled at regular intervals to $Q$ samples. $\alpha$ is the bandwidth compression factor, taking a value between $[0,1]$, hence saving $(1-\alpha) \times 100 \%$ from the originally occupied bandwidth. Obviously, in OFDM $\alpha$ is unity.

\section{B. Correlation Matrix \& ICI}

SEFDM's SE gain is obtained by compressing the frequency spacing between subcarriers; thus each subcarrier is purposely interfered with every other subcarrier resulting in ICI, as illustrated in Fig.1. Unlike OFDM, where ICI is ideally zero and there is no cross correlation between the subcarriers, in SEFDM there is finite cross correlation, where the values may be obtained to have complete description of the signal properties and allow mitigation of the ICI effects. For an

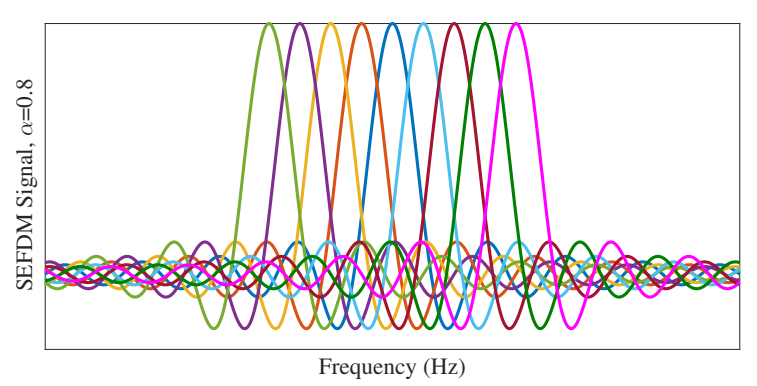

Fig. 1: SEFDM spectrum for $\alpha=0.8$.

SEFDM symbol of length $N$, the cross correlation between two subcarriers $m$ and $n$ is given by [5]

$$
\begin{aligned}
c(m, n) & =\frac{1}{Q} \sum_{k=0}^{Q-1} \exp \left(\frac{j 2 \pi m \alpha k}{Q}\right) \exp \left(\frac{-j 2 \pi n \alpha k}{Q}\right) \\
& =\frac{1}{Q}\left[\frac{1-\exp (j 2 \pi \alpha(m-n))}{1-\exp \left(\frac{j 2 \pi \alpha(m-n)}{Q}\right)}\right]
\end{aligned}
$$

This equation calculates entries of the cross correlation matrix C of size $N \times N$, where the rows are for $n=0, \ldots, N-1$ and columns for $m=0, \ldots, N-1$. When $m=n$, the cross correlation is the same as auto correlation and it is equal to one, thus, the matrix $\mathbf{C}$ has a diagonal of ones, while the non-diagonal components symbolize the correlation among subcarriers. For a detailed mathematical treatment refer to [16].

\section{APPROACH I: ENCODED SEFDM}

\section{A. System Model}

The block diagram of the proposed system is shown in Fig. 2. For proof of concept, we initially consider a system only impaired by additive white Gaussian noise (AWGN) and then extend the treatment into SEFDM operating in a static multipath frequency selective channel.

The model uses an SEFDM transmitter similar to that reported in [17], where standard turbo coding was used with no interference cancellation and with receiver hard demapping. Conversely, in this work, block, convolutional and turbo channel coding methods are applied at the transmitter with soft demapping at the receiver. Unless otherwise stated, the results in this section are for the standard parameters shown in Table.1.

In this study a technique inspired by that of [18] is followed for temporal channel equalization, which is done before the receiver FFT, by utilizing a linear minimum mean square error (MMSE) equalizer. The MMSE equalizer reverses the channel distortion through the application of a linear filter to the received signal time samples $\mathbf{y}_{i}$. The filter coefficients are determined according to MMSE criterion using

$$
\mathbf{G}_{\mathrm{MMSE}}=\mathbf{H}^{-\mathbf{1}}\left(\mathbf{I}_{\mathbf{Q}}+\sigma_{\mathbf{n}}^{2} \mathbf{I}_{\mathbf{Q}}\right)^{-\mathbf{1}},
$$

and yielding the output simply expressed in

$$
\mathbf{y}_{0}=\mathbf{G}_{\mathrm{MMSE}} \times \mathbf{y}_{\mathbf{i}}
$$


TABLE I: SEFDM system parameters

\begin{tabular}{|c|c|}
\hline Modulation & 4-QAM(Quadrature Amplitude Modulation) \\
\hline Turbo encoder & $\begin{array}{c}\left.(5,7,3), R_{c}=1 / 3 \text { with zero biting( } 8 \text { bits }\right), \text { Interleaver size } 2048, \text { and output block size } \\
(2048 \times 3+8)=6152\end{array}$ \\
\hline Turbo encoder with puncturing & $\begin{array}{l}\left.(5,7,3), R_{c}=1 / 2 \text { with zero biting( } 8 \text { bits }\right), \text { Interleaver size } 2048, \text { and output block size } \\
(2048 \times 2+8)=4104\end{array}$ \\
\hline Recursive systematic convolutional coder (RSCC) & $(5,7,3), R_{c}=1 / 2$ and external interleaver of size 2048 \\
\hline Systematic Reed-Solomon(RS) block code & $(223,255,32), R_{c}=223 / 255$ \\
\hline Serial concatenation channel coding (SCCC) & outer RS $(223,255,32)$, internal turbo, $R_{c}=\frac{223}{255} \times \frac{1}{3}=\frac{223}{765}$ \\
\hline Turbo decoder & Log-MAP decoder \\
\hline Turbo decoder iterations & 8 \\
\hline Convolutional decoder & Viterbi Algorithm (VA) \\
\hline SEFDM Symbol Size(N) & 16 \\
\hline Channel(I) & Additive white Gaussian noise (AWGN) \\
\hline Channel(II) & $h(t)=0.8765 \delta(t)-0.2279 \delta\left(t-T_{s}\right)+0.1315 \delta\left(t-5 T_{s}\right)-0.4032 \exp \left(\frac{i \pi}{2}\right) \delta\left(t-7 T_{s}\right)$ \\
\hline
\end{tabular}

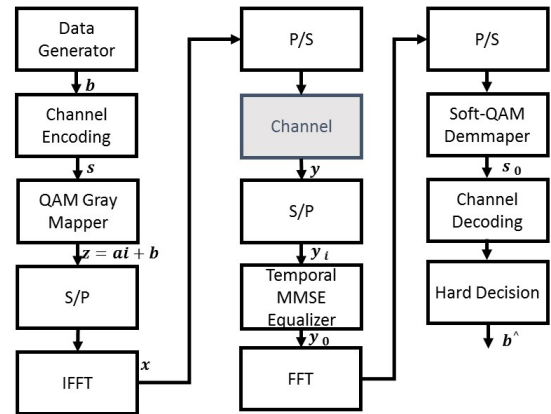

Fig. 2: Approach I transceiver block diagram.

where $\mathbf{H}$ is the $Q \times(Q+L-1)$ channel matrix of a multipath channel of length $L, \mathbf{I}_{Q}$ is an identity matrix of size $Q \times Q$, $Q$ is the number of samples in one SEFDM symbol and $\sigma_{n}{ }^{2}$ is the noise variance.

Thereafter, an FFT acting as a demodulator, transfers the time samples $\mathbf{y}_{0}$ back into the frequency domain, where they are fed to the soft-QAM demmaper. Soft demapping is chosen because it outperforms hard decision methods especially when there is serious signal degradation such as that encountered in SEFDM with higher ICI levels than those of OFDM. The soft bits $\mathbf{s}_{0}$ are fed to the decoder to get an estimate $\hat{\mathbf{b}}$ of the transmitted bits. The next section analyzes the effect of several system parameters by examination of the BER performance and SE.

\section{B. Results}

For coded SEFDM, The effective spectral efficiency SE, measured in $b / s / H z$, is expressed in terms of the compression factor $\alpha$, the modulation constellation size $M$, the code rate $R_{c}$ and the occupied bandwidth $W$ in $\mathrm{Hz}$ as in

$$
\mathrm{SE}=\frac{R_{c} \times \log _{2}(M)}{\alpha \times W}
$$

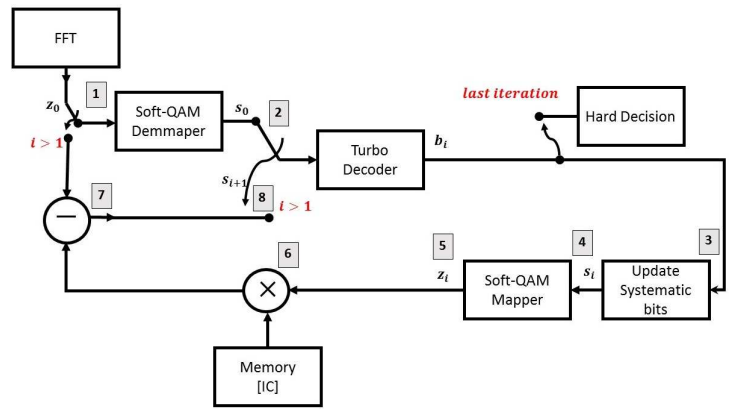

Fig. 3: Approach II receiver symbol detection process diagram.

The related spectral efficiency gain $\zeta$ (relative to OFDM) is simply defined as

$$
\begin{aligned}
\zeta & =\frac{\mathrm{SE}_{\mathrm{SEFDM}}-\mathrm{SE}_{\mathrm{OFDM}}}{\mathrm{SE}_{\mathrm{OFDM}}} \times 100 \% \\
& =\left(\frac{1}{\alpha}-1\right) \times 100 \% .
\end{aligned}
$$

In this section we use the above formula for assessing gains achieved by different coded SEFDM systems with varying values of $\alpha$. Fig. 4 shows $\zeta$ versus $\alpha$; a higher SE improvement is gained for lower $\alpha$, the maximum shown here is $67 \%$ when $\alpha=0.6$.

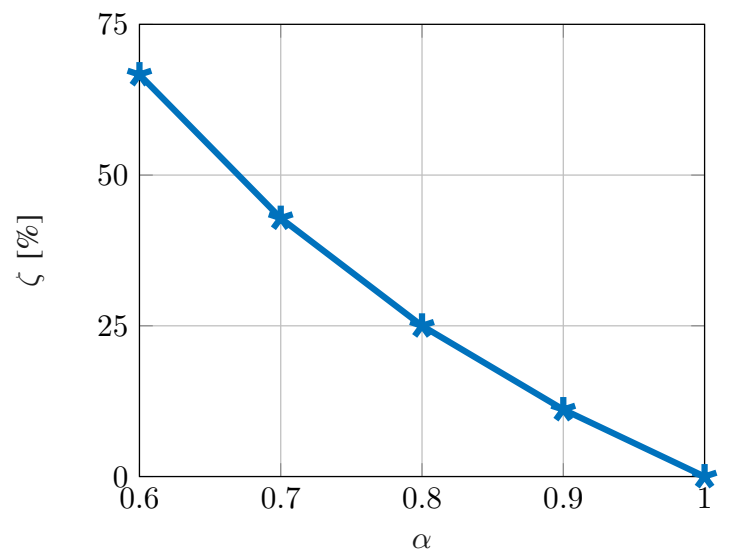

Fig. 4: SEFDM SE improvement percentage $\zeta$ vs. $\alpha$. 
Convolutional coding with interference cancellation and turbo equalization has been successfully utilized with SEFDM and reported in [11]. Using capacity approaching forward error correction techniques, such as turbo coding, is expected to yield advantages, especially in multipath channels. In this section, different channel codes are explored without interference cancellation. Recursive systematic convolutional code (RSCC) rate (1/2); Reed-Solomon (RS) block code $(223,255,32)$; turbo code of rates $(1 / 2)$ and $(1 / 3)$ and the serial concatenation of RS with turbo code. System parameters are listed in Table.1.

Fig. 5(a-e), shows results of modeling in AWGN channels in terms of error rates versus $E_{b} / N_{0}$. In each of the plots, OFDM is used as a reference to indicate the utility of a particular coding method for the SEFDM cases. The results of Fig. 5(a) clearly show the inability of block coding to correct for errors, largely created by interference, which is particularly evident in higher $E_{b} / N_{0}$ regions and for higher spectral efficiency gains (lower $\alpha$ ). Convolutional coding Fig. 5(b) fares better, but is still unable to offer good performance for low $\alpha$ values. Turbo coding, as expected, performs better and its performance improves with the reduction of the coding rate from $(1 / 2)$ to $(1 / 3)$ shown in Fig. 5(c) and Fig. 5(d), respectively.

The advantage of using the MMSE channel equalization and the soft demapping is evident when the results of this work are compared to our earlier work in [17], where turbo coding was used with simple zero forcing detection. The results of using rate $(1 / 3)$ indicate that for $\alpha=0.6$, a spectral efficiency gain of $67 \%$ can be obtained at the expense of close to $3 \mathrm{~dB}$ error penalty when 8 turbo iterations are used. A series of other tests were carried out where serially concatenated RS-Turbo coding was applied, however, the improvement over turbo coding was limited as is shown in Fig. 5(e).

The same coding methods were applied to SEFDM signals propagating through a frequency selective channel (Channel II in the table) under the assumption of both perfect synchronization and perfect channel estimation with a sufficient cyclic prefix that totally eliminates ISI. Only the results of $(1 / 3)$ turbo coded signals are reported here in Fig. 5(f) and as expected the performance degrades relative to the AWGN case for both OFDM and SEFDM, while the performance gap also degrades for low $\alpha$.

We conclude that although coding improves the error performance in SEFDM, as it indeed does for OFDM systems, there is a serious limitation, which is ICI. Therefore, further improvements in spectral efficiency and the reduction in the number of turbo iterations would require better removal of the interference from the received signal. This has led to the development of a new type of SEFDM receivers with interference cancellation as discussed in the section below.

\section{APPROACH II: ITERATIVE ICI CANCELLATION}

In this approach, after the receiver FFT, an iterative canceler is implemented to mitigate the effect of ICI, where soft information circulates through over a number of iterations and at the last iteration a hard decision is taken. The transmitter side is identical to the one of approach I shown in Fig. 2, however, the receiver side SEFDM symbol detection implementation is shown in Fig. 3 with its steps being enumerated for ease of reference. The operation of the receiver is detailed below.

\section{A. System Model}

Starting from the top left of the receiver block diagram and assuming the only impairment is white noise, given by the vector $\mathbf{n}$, the initial estimate vector $\mathbf{z}_{0}$ is

$$
\mathbf{z}_{\mathbf{0}}=\mathbf{C z}+\mathbf{n}
$$

where $\mathbf{C}$ is the correlation matrix and $\mathbf{z}$ is the transmitted complex symbols vector.

The design philosophy of this receiver is based on an iterative process, which may be viewed as a synthesis of the processes used for MIMO in [14] and for convolutional coded SEFDM in [11]. In this work, the turbo decoder ouputs soft systematic bits, which are used to generate, through a soft mapping and ICI estimation processes, approximate "replicas" of received symbols, which in turn are used to cancel the interference, iteratively. At the first iteration, the turbo decoder is fed by soft bits $\mathbf{s}_{0}$ from the soft-QAM demapper. There are two outputs from the turbo decoder; the soft systematic bits $\mathbf{b}_{i}$ used to update the encoded stream shown in the figure and the extrinsic LLR information (inside the turbo decoder block and therefore not shown explicitly in the figure) that will be fed to the turbo decoder as $a$-priori information in the next iteration. The updated encoded stream $\mathbf{s}_{i}$ is mapped again via soft-QAM mapper to get the $i^{\text {th }}$ estimate of the transmitted symbols $\mathbf{z}_{i}$.

The non-diagonal elements of cross correlation matrix $\mathbf{C}$ mentioned earlier in (2) represent the ICI between subcarriers in SEFDM systems. Therefore, by setting the diagonal to zeros using

$$
\mathbf{I C}=\mathbf{C}-\operatorname{diag}(N, N)
$$

where $\operatorname{diag}(N, N)$ is an $(N \times N)$ identity matrix. The resulting interference canceler matrix IC is then multiplied by the estimated vector symbols $\mathbf{z}_{i}$ to evaluate the estimated ICI, given by the term $\left(\mathbf{I C} \times \mathbf{z}_{i}\right)$. The estimated ICI is subtracted from the initial estimate vector $\mathbf{z}_{0}$, as stated in (9), to give $\mathbf{s}_{i+1}$, which forms the input to the next iteration

$$
\begin{aligned}
\mathbf{s}_{\mathbf{i}+\mathbf{1}} & =\mathbf{z}_{\mathbf{0}}-\mathbf{I} \mathbf{C} \times \mathbf{z}_{\mathbf{i}} \\
& =\mathbf{C}\left(\mathbf{z}_{\mathbf{0}}-\mathbf{z}_{\mathbf{i}}\right)+\mathbf{z}_{\mathbf{i}}+\mathbf{n} .
\end{aligned}
$$

\section{B. Results}

The results shown in Fig. 6 are for the same system specifications of approach I, turbo code rate (1/3) but with only 2 iterations for the turbo decoder instead of 8 . Three interference cancellation iterations are used and it was observed that no further enhancement in system performance can be achieved for a higher number of iterations, since the value of estimated symbols will be very close to those of the originally transmitted symbols after two or three iterations. Therefore, the element 


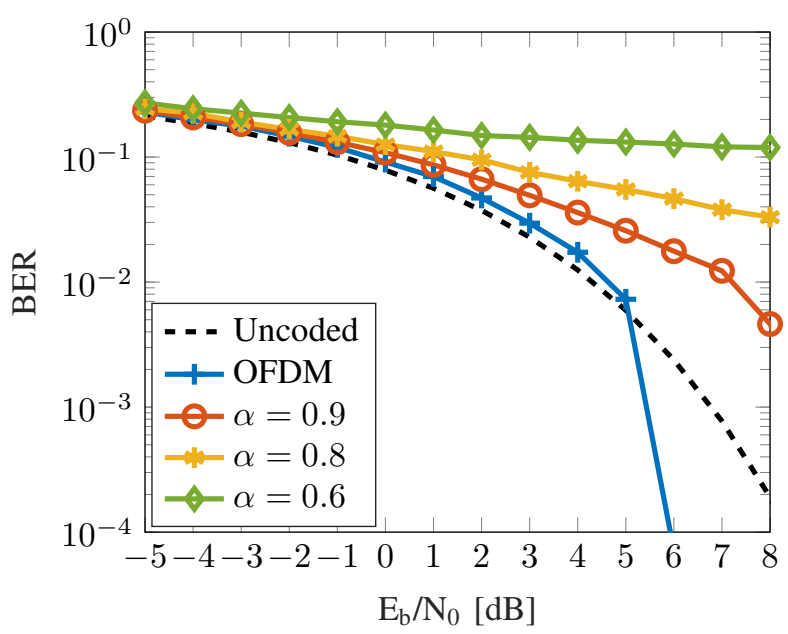

(a) RS coding, AWGN channel

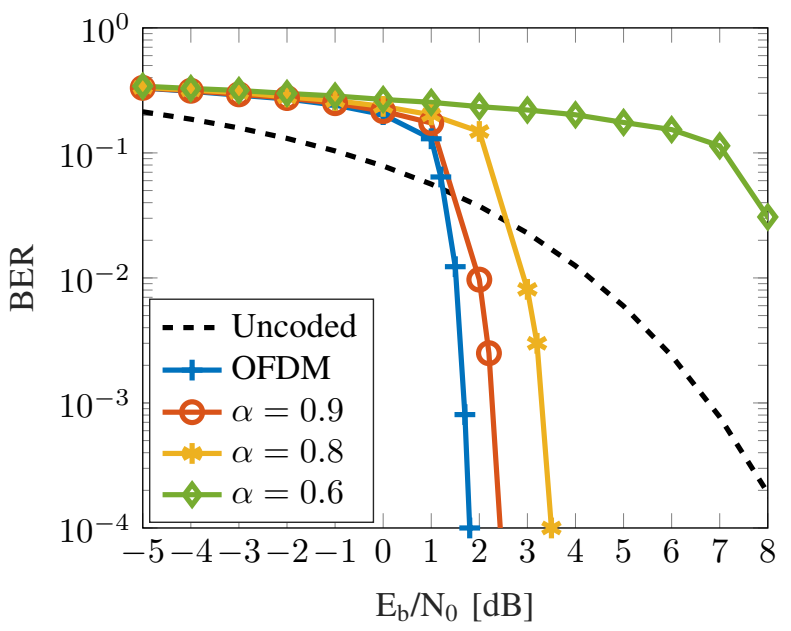

(c) Turbo coding $\left(R_{c}=1 / 2\right)$, AWGN channel

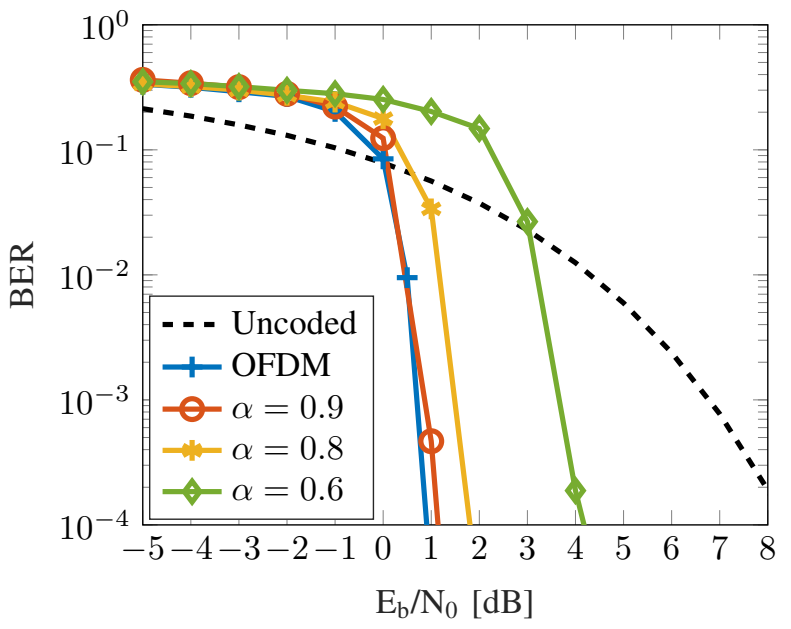

(e) SCCC, AWGN channel

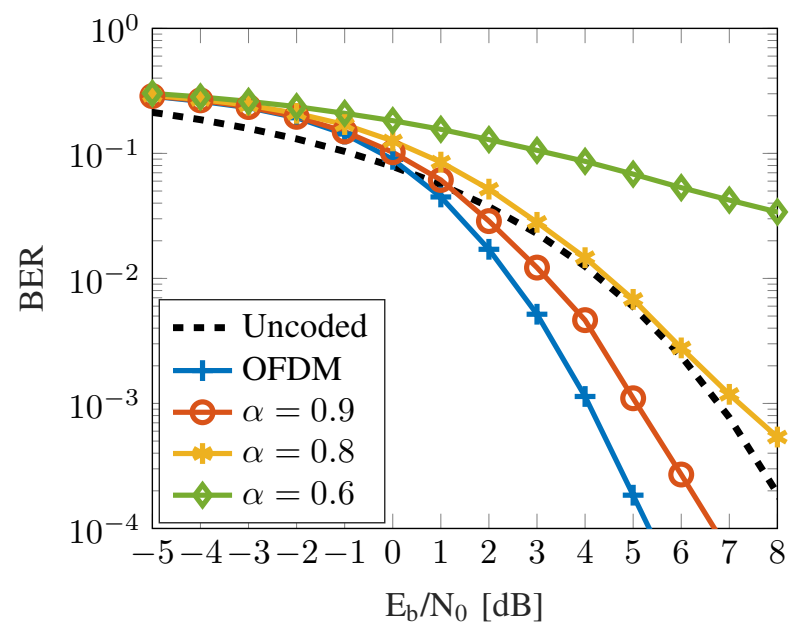

(b) Convolutional Coding, AWGN channel

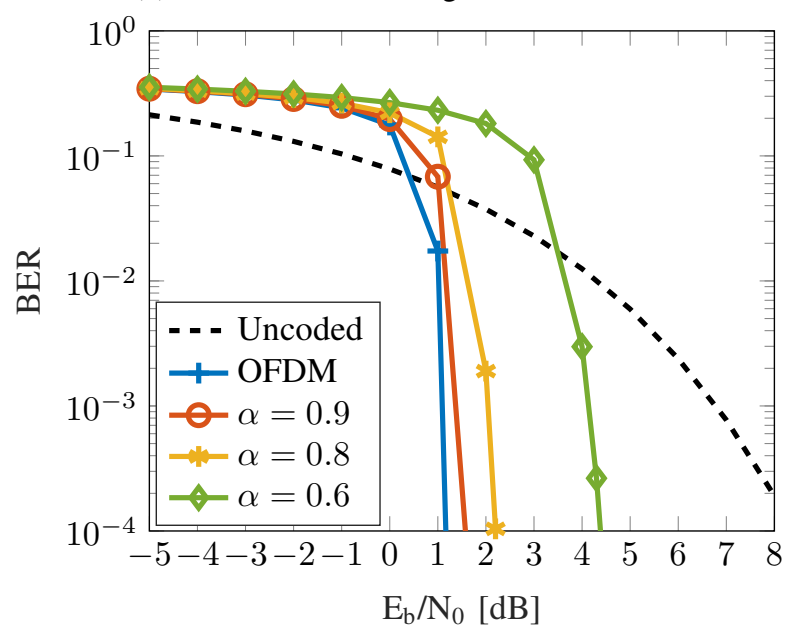

(d) Turbo coding $\left(R_{c}=1 / 3\right)$, AWGN channel

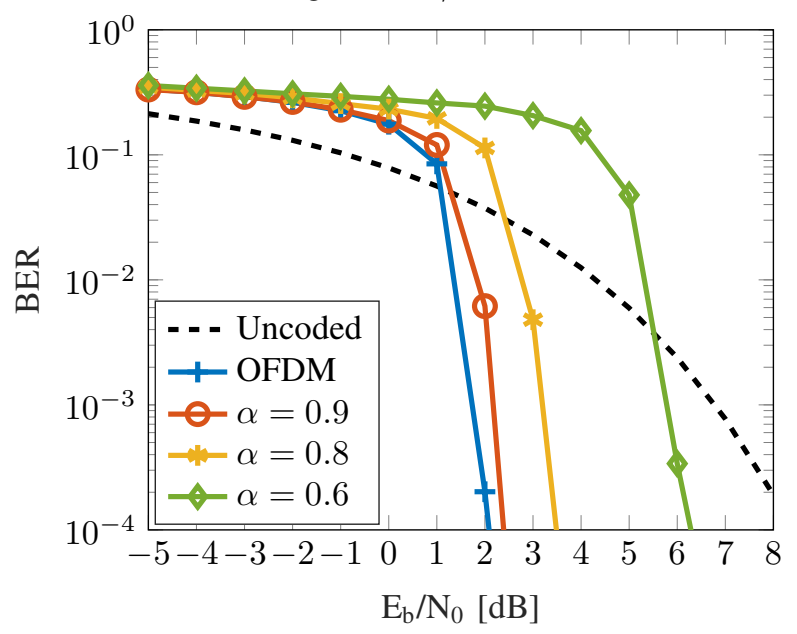

(f) Turbo coding $\left(R_{c}=1 / 3\right)$, multipath channel

Fig. 5: Approach I:SEFDM system 4-QAM BER performance with different coding algorithms, for $\mathrm{N}=16$. 


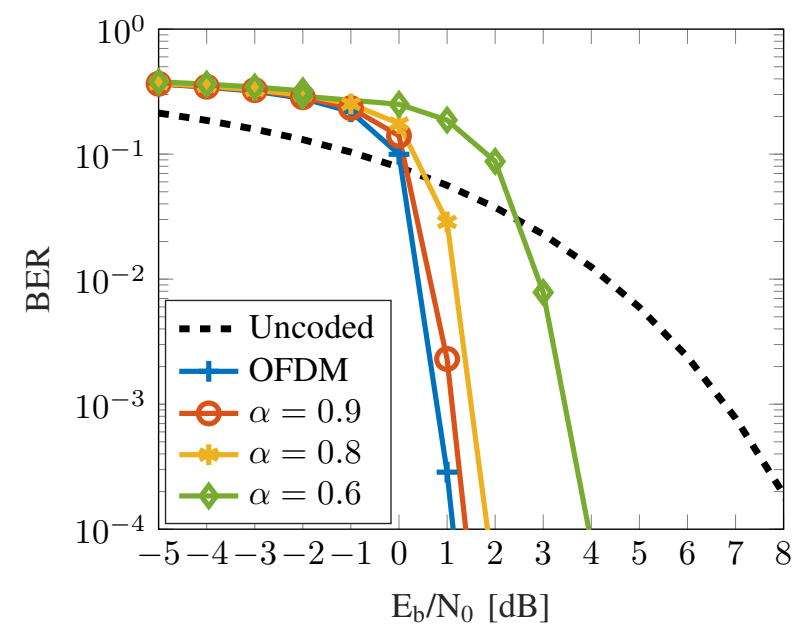

Fig. 6: Approach II: Turbo coding (1/3), 4-QAM BER performance for $\mathrm{N}=16$ over AWGN channel.

$\mathbf{C}\left(\mathbf{z}_{0}-\mathbf{z}_{i}\right)$ in (9) will maintain the same estimate. This relatively low number of iterations results in superior performance to that of Fig. 5(d), where for the same code used, a higher number of iterations was necessary to obtain acceptable performance.

The results show the efficiency of this interference cancellation technique and the effectiveness of turbo coded SEFDM in obtaining spectral efficiency gains up to $67 \%$ with a power penalty of $2.8 \mathrm{~dB}$.

\section{Conclusions}

In this work, we presented the basics of coded SEFDM systems, where different FEC coding techniques were applied to enhance SEFDM error rates in AWGN and frequency selective channels. A new receiver architecture, with MMSE equalization and soft demapping, was tested using a set of simulation based experiments, applying block, convolutional and turbo coding at two different rates. The advantages of the proposed receiver architecture over previously published work were evident from BER results which showed the operation of SEFDM to result in spectral efficiency gain up to $67 \%$ with limited power penalty, when compared to OFDM systems. Notwithstanding, the operation required a relatively high number of turbo iterations and was still limited by the ICI resulting from the non-orthogonal nature of SEFDM. To ameliorate the effect of such ICI, this work proposed and tested a new parallel interference cancellation receiver that operates iteratively over the received SEFDM symbols and improves the performance of coded systems. In this work, we reported the application of this receiver to turbo coded SEFDM and show improvement of error performance with a small number of iterations. For the interference cancellation receiver, when turbo coding was used, spectral efficiency gain of $67 \%$ was achieved at the expense of $2.8 \mathrm{~dB}$ of power penalty relative to an otherwise equivalent OFDM system. Hence, the proposed system and SEFDM signals offer key spectral efficiency advantage over many of the other signal formats proposed for $5 \mathrm{G}$, at the expense of additional receiver complexity and potentially added latency due to the iterations. Refinement of the receiver structure is the subject of ongoing work and it is envisaged that the signals and architectures proposed here offer alternative approaches for the implementation of wireless systems where spectrum is scarce, such as $5 \mathrm{G}$ systems and beyond.

\section{ACKNOWLEDGMENT}

We are grateful to UCL for funding Hedaia Ghannam's PhD studies through the Overseas Research Student Award (ORS) and the UCL Dean of Engineering Sciences Award.

\section{REFERENCES}

[1] F. Luo and C. Zhang, Signal Processing for 5G: Algorithms and Implementations, ser. Wiley - IEEE. Wiley, 2016.

[2] P. Banelli, S. Buzzi, G. Colavolpe, A. Modenini, F. Rusek, and A. Ugolini, "Modulation formats and waveforms for 5G networks: Who will be the heir of OFDM?: An overview of alternative modulation schemes for improved spectral efficiency," IEEE Signal Process. Mag., vol. 31, no. 6, pp. 80-93, Nov. 2014.

[3] T. Hwang, C. Yang, G. Wu, S. Li, and G. Y. Li, "OFDM and its wireless applications: A survey," IEEE Trans. Veh. Technol., vol. 58, no. 4, pp. 1673-1694, May 2009.

[4] M. Rodrigues and I. Darwazeh, "A spectrally efficient frequency division multiplexing based communications system," in Proc. 8th Int. OFDM Workshop, Hamburg, 2003, pp. 48-49.

[5] P. N. Whatmough, M. R. Perrett, S. Isam, and I. Darwazeh, "VLSI architecture for a reconfigurable spectrally efficient FDM baseband transmitter," IEEE Trans. Circuits Syst. I, Reg. Papers, vol. 59, no. 5, pp. 1107-1118, May 2012.

[6] I. Kanaras, A. Chorti, M. R. D. Rodrigues, and I. Darwazeh, "Spectrally efficient FDM signals: Bandwidth gain at the expense of receiver complexity," in Proc. IEEE Int. Conf. Commun. (ICC), June 2009, pp. $1-6$.

[7] T. Xu and I. Darwazeh, "Transmission experiment of bandwidth compressed carrier aggregation in a realistic fading channel," IEEE Trans. Veh. Technol., accepted 2016.

[8] F. Rusek and J. Anderson, "Multistream faster than Nyquist signaling," IEEE Trans. Commun., vol. 57, no. 5, pp. 1329-1340, May 2009.

[9] X. Zhang, L. Chen, J. Qiu, and J. Abdoli, "On the waveform for 5G," IEEE Commun. Mag., vol. 54, no. 11, pp. 74-80, Nov. 2016.

[10] C. Kim, Y. H. Yun, K. Kim, and J. Y. Seol, "Introduction to QAMFBMC: From waveform optimization to system design," IEEE Commun. Mag., vol. 54, no. 11, pp. 66-73, Nov. 2016.

[11] T. Xu, S. Mikroulis, J. E. Mitchell, and I. Darwazeh, "Bandwidth compressed waveform for $60-\mathrm{GHz}$ millimeter-wave radio over fiber experiment," J. of Lightwave Technol., vol. 34, no. 14, pp. 3458-3465, July 2016.

[12] Y. Zhao and S. G. Haggman, "Intercarrier interference self-cancellation scheme for OFDM mobile communication systems," IEEE Trans. Commun., vol. 49, no. 7, pp. 1185-1191, July 2001.

[13] S. Trautmann, T. Karp, and N. J. Fliege, "Frequency domain equalization of DMT/OFDM systems with insufficient guard interval," in Proc. IEEE Int. Conf. Commun. (ICC), vol. 3, May 2002, pp. 1646-1650 vol.3.

[14] A. F. Molisch, M. Toeltsch, and S. Vermani, "Iterative methods for cancellation of intercarrier interference in OFDM systems," IEEE Trans. Veh. Technol., vol. 56, no. 4, pp. 2158-2167, July 2007.

[15] T. Xu and I. Darwazeh, "A soft detector for spectrally efficient systems with non-orthogonal overlapped sub-carriers," IEEE Communications Letters, vol. 18, no. 10, pp. 1847-1850, Oct 2014.

[16] R. Clegg, S. Isam, I. Kanaras, and I. Darwazeh, "A practical system for improved efficiency in frequency division multiplexed wireless networks," IET Commun., vol. 6, no. 4, pp. 449-457, Mar. 2012.

[17] H. Ghannam and I. Darwazeh, "Comparison of turbo decoder and turbo equalizer for spectrally efficient FDM system," in Proc. 10th Int. Symp. Commun. Syst. Networks and Digital Signal Process. (CSNDSP), July 2016, pp. 1-6.

[18] M. Tuchler, A. C. Singer, and R. Koetter, "Minimum mean squared error equalization using a priori information," IEEE Trans. Signal Process., vol. 50, no. 3, pp. 673-683, Mar. 2002. 